\title{
El título como recurso didáctico
}

\author{
Jose Antonio León y Angel Martín
}

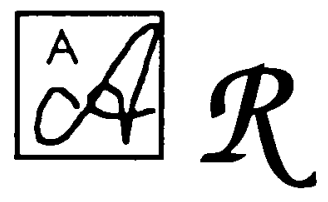

Se revisan aqui las funciones que tiene el titulo de un texto y se propone un modelo de "instrucción razonada" en tres fases para que el adiestramiento en titulación constituya una nueva vía para mejorar la comprensión lectora.

\section{INTRODUCCION}

Probablemente una de las tareas más delicadas en la que se encuentre un escritor sea la de culminar su trabajo con un título que represente dignamente lo escrito. Si comenzó con una idea inicial, es muy posible que en la medida en que el escrito avanza, esa idea se haya ido modificando paulatinamente, al compás de los nuevos añadidos. Sólo al final de ese proceso es donde el encabezamiento adquiere todo su sentido representativo. Una vez alcanzada esta pretensión, el título cumple otra función esencial, la de establecer contacto con el exterior. Como primicia de la información que representa, orienta al lector sobre su contenido facilitando, en caso de interesar a éste, la iniciación de la estrecha relación entre el escritor y lector. Quizás por ello, los medios de comunicación dedican buena parte de su tiempo a la búsqueda de títulos o encabezamientos que resulten sugerentes para el lector potencial. Su presencia, mayoritaria en un medio escrito como la prensa, ha ido calando de manera progresiva en otros medios de comunicación como la radio y televisión. A este respecto puede observarse como en algunos de los telediarios que se emiten actualmente, la noticia que va a ser tratada va precedida de un encabezamiento o título que se presenta bajo un doble formato, leído por el presentador y escrito en pantalla, para que el televidente lo oiga y lea simultáneamente. No menos impor- 


\section{0}

tante es su presencia en el mundo educativo. Frecuentemente la información que aparece en los libros de textos e informes científicos suele ir precedida de títulos, apartados y subapartados que orientan al lector sobre las diferentes partes del escrito.

Recientemente el título ha sido estudiado desde un punto de vista científico. Entre otras disciplinas, la psicología viene interesándose por su estudio desde hace tan sólo dos décadas. Durante este tiempo, su investigación se ha orientando fundamentalmente hacia las repercusiones que el título ejerce sobre la comprensión y retención del material leído. Desde su punto de vista ha tratado o esta tratando de responder a preguntas sobre el concepto mismo de título y de sus características, sobre su utilidad, sobre los procesos y estructuras implicados en su procesamiento o sobre cómo se realiza una abstracción de la información referente. Aún más reciente es el interés hacia aspectos relacionados con las estrategias de los lectores y su utilidad como herramienta didáctica en el aula. Desde la perspectiva psicológica el título se asume como un tipo de encabezamiento que precede a la información que va a ser presentada inmediatamente después. Pero a diferencia de otras acepciones (e.g. titular), el título representa el núcleo de la información relevante, corresponde al enunciado más representativo sobre el tópico leído. Debe observarse que, aunque el título trata de recoger la idea nuclear sobre la que pivotan el resto de las ideas expresadas en el texto, no debe confundirse con un esquema o un resumen. Aunque en todos estos casos se selecciona fundamentalmente la información relativa al tema central del pasaje, su extensión se reduce a unas pocas palabras, a lo sumo una frase.

En algunos casos, la investigación psicológica sobre el título se ha asociado a factores textuales, como la complejidad y ambigüedad del mismo. En estas situaciones, el estudio se ha dirigido a evaluar el posible efecto que su simple presencia ejerce al despejar o atenuar la complejidad o imprecisión del contenido del pasaje. En otros casos, su interés se ha encauzado hacia aspectos más relacionados con las características del lector, como el nivel de sus conocimientos previos sobre el tema o tópico del texto. Sin embargo y a pesar del interés e importancia que se otorga a los títulos en la comprensión lectora, no se ha prestado una excesiva atención a las posibles implicaciones educativas que estos estudios sugieren. Es probable que una de las razones por la que se produjese este hecho se deba a la escasa conexión entre trabajos teóricos y aplicados. No suele ser frecuente que un investigador interesado en aspectos básicos de investigación disponga de tiempo e interés en aplicarlo dentro de un contexto educativo. Nuestra pretensión en este trabajo hace referencia a estos dos aspectos. Por un lado, presentaremos de manera sucinta algunos de los trabajos teóricos más representativos. Por otro, nos centraremos en cómo estos conocimientos teóricos sobre el título pueden ser aplicados en el aula como una actividad válida y complementaria para los diferentes contenidos curriculares que se imparten en cualquier nivel educativo. 


\section{EL TITULO COMO ORGANIZADOR DE LA INFORMACION IEIDA}

Como ya hemos comentado, la mayor parte de los trabajos realizados sobre los efectos del título en la comprensión lectora y su retención provienen de la psicología, fundamentalmente de su orientación cognitiva. Dentro de esta perspectiva se asume la idea general de que, si una determinada información va precedida de un título que represente dicho contenido, producirá una mejora considerable en la comprensión de esa información leída por parte del sujeto, que si ese mismo contenido se lee sin el título. Varias han sido las explicaciones que han dado cuenta de este fenómeno. Una muy reciente alude a las características físicas en las que se enmarca el título. Proviene de la teoría de la función visual (Grant y Davey, 1991) y se basa en los atributos espaciales de la memoria. Según esta concepción, los lectores pueden hacer uso de los diferentes espacios que aparecen en los textos (espacios en blanco, espacios entre párrafos..) como indicadores útiles para codificar los segmentos de información. Trasladando ésta explicación al estudio de los títulos, los espacios usuales en los que se ubica el encabezamiento del texto, podrían interpretarse como una señalización, una llamada de atención que facilita la codificación de la información del título y, de esa manera, preparar al lector sobre lo que vendrá a continuación.

Pero como es sabido, la explicación más ligada a este punto de vista teórico ha sido la teoría del esquema. Una característica básica de esta teoría es la de considerar que la información que procesamos y almacenamos en nuestra memoria se realiza a través de "paquetes de información" altamente organizados y jerarquizados en nuestra mente a los que denominamos esquemas y subesquemas. Un esquema mental puede considerarse como una representación genérica que, almacenado en la memoria, corresponde a un objeto, idea, concepto o proceso que contiene ciertos atributos o expectativas acerca de lo que representa. Así, por ejemplo, el esquema "cara" contiene variables que corresponden a los elementos esperados dentro del concepto (e.g. nariz, ojos, boca, mejillas...). Todos estos elementos surgen de manera instantánea una vez activado el esquema "cara". Incluso cada una de estas variables del esquema pueden ser subesquemas de aquel (e.g. boca), y conformar asimismo otra serie de valores que pueden emerger al activar dicho subesquema (e.g. lengua, dientes, labios, encías...).

Una asunción que subyace a las explicaciones de esta teoría para explicar cómo procesamos la información escrita hace referencia a que la información previa, la información ya almacenada en nuestra memoria y representada en esquemas mentales, proporciona un apoyo seguro y fiable a la nueva información entrante. Pero puede ocurrir que, aunque poseamos un conocimiento previo adecuado y exista una fuerte referencia lógica entre las frases que componen el texto, el párrafo leído no sea suficiente para evocarnos la situación, objeto o tema descrito en el pasaje. En estos casos, el simple hecho de proporcionar un título puede ofrecer un aspecto clarificador, bien haciendo que recobre el sentido global, bien organizando y coordinando lo que hasta entonces era un desorden. Sírvanos como ejemplo el pasaje elaborado por Dooling y Lachman (1971): 
"Con las joyas empeñadas para financiarle, nuestro beroe bravamente desafiaba las risas despectivas que intentaban persuadirle de su objetivo. "Tus ojos te engañan" decian. "Un buevo y no una mesa representa más fielmente este planeta inexplorado", argumentó. Entonces las tres robustas bermanas se dispusieron a la búsqueda de pruebas. Durante su esfuerzo, unas veces sobre una calma inmensa y otras más frecuentes sobre turbulentas cimas y valles, los dias se bacian semanas y una duda temerosa se cernía sobre su límite. Por fin, en ninguna parte, criaturas aladas aparecieron dando la bienvenida significando el exito transcendental".

Aunque el texto nos resulte familiar por la gran cantidad de acontecimientos que hemos vivido recientemente relacionados con este hecho, no resultaba tan familiar veinte años atrás. Tal y como se esperaba en este trabajo, el pasaje resultó difícil de recordar. El escrito había sido redactado de una manera intencionadamente ambigua y general que hacía difícil su comprensión. Sin embargo, cuando a uno de los dos grupos de sujetos que participaron se les presentó previamente el título a su lectura ("Cristobal Colón descubre América"), el recuerdo de este grupo mejoró ostensiblemente. Sin el título, la presencia de metáforas hacía que el texto fuera virtualmente ininteligible, dando la sensación como si los sujetos "no supieran qué hacer ni decidir con la información leída". Con este trabajo, Dooling y Lachman demostraron que el hecho de proporcionar un título a los sujetos inmediatamente antes de leer un texto, incrementaba significativamente el recuerdo de las palabras y frases del texto, sobre todo de aquéllas que tenían alguna relación semántica con el título proporcionado, por lo que ellos supusieron que la información temática transmitida con el título, activaría esquemas capaces de representar el significado central del texto, esquemas que, a su vez, eran utilizados en la reconstrucción de la información.

Esta línea de trabajos fue seguida con una metodología parecida por Bransford y cols. en los inicios de los años setenta (e.g. Bransford y Johnson, 1972). En una serie de trabajos variaron la disposición del conocimiento previo necesario para la comprensión, proporcionando a algunos lectores el título apropiado antes de leer el material escrito, inmediatamente después de su lectura en otros o privando a los lectores de la presencia del título. Según estos autores, para que se produzca una comprensión coherente del texto, no basta con poseer un conocimiento previo adecuado, sino que tal conocimiento debe ser activado o evocado con anterioridad a la lectura o, al menos, durante el proceso lector. Sin embargo, trabajos posteriores han revelado que en situaciones en las que la presentación del título se realiza con posterioridad a la lectura también produce una influencia positiva, denominada influencia retroactiva. Estos resultados también se vieron complementados con los obtenidos en los trabajos de Anderson y cols., (Anderson y Pichert, 1978) en los que también se utilizaron textos deliberadamente ambiguos capaces de generar dos versiones distintas y equiprobables. En sus estudios también incluyeron a varios sujetos de distinta procedencia cultural y social. Los resultados obtenidos indicaron una estre- 
cha relación entre la procedencia social y cultural y la versión recorda$\mathrm{da}$, influyendo, por tanto, la perspectiva social del lector.

Trabajos como los ya citados siguieron realizándose en años sucesivos, fortaleciendo estos resultados. Actualmente se asume que, si los esquemas del sujeto están relacionados con la información del título, éste puede influir positivamente en su posterior procesamiento del texto. Tal y como señalan algunos autores más recientes, en tales casos los títulos proporcionan indicios que activan el conocimiento previo y relevante del sujeto sobre un tema dado (Wilhite, 1988), acentúan las relaciones entre conceptos y hechos en el pasaje leído (Spydirakis y Standal, 1987), proporcionan pistas sobre el contenido para la posterior recuperación de la información leída ante tareas de recuerdo y alertan al lector hacia la estructura de alto nivel, es decir, hacia las partes donde se encuentran las ideas principales del pasaje leído (Kozminsky, 1977).

En general, todos estos estudios confirman el hecho de que el lector debe construir el significado, integrando lo que ya conoce con la nueva información y esa labor de integración puede ser facilitada de manera notable si al lector se le proporciona explícitamente un contexto apropiado, unos indicios adecuados. Ello parece producirse porque el contexto activa el esquema superior que orienta el procesamiento del texto, eliminando su ambigüedad. De esta manera, la teoría del esquema concibe la comprensión de textos como un proceso de comprobación de hipótesis. Cuando el lector inicia su lectura o cuando se le proporciona un contexto a través de un título, le permite generar determinados esquemas-hipótesis sobre los que descansa el sentido de lo que está leyendo, pero, como es lógico, estas hipótesis han de ser constantemente contrastadas por la información que llega del texto. Si no se establece discordancia entre las hipótesis y los datos, tal interpretación se mantendría y se haría consistente si la confirmación se lleva a efecto. Pero en el caso en que la información leída no coincidiera con los esquemas, la interpretación se haría inviable y llevaría a cabo otras que trataran de concordar con los nuevos datos.

Explicaciones como éstas aluden a algunos tipos de problemas importantes en la comunicación del material escrito y en su proyección educativa, como es la trascendencia que tiene el hecho de que el lector posea previamente un conocimiento adecuado a la tarea encomendada o la importancia que tiene el ofrecer claves en el texto a fin de facilitarle una interpretación correcta de la información leída. El primer punto se confirma en un trabajo reciente (Wilhite, 1989). Los resultados de este estudio limitaban el efecto del título sólo a lectores que poseían un nivel relativamente alto de conocimientos previos sobre el tema. Este hecho resalta la importancia del conocimiento previo y explica, al menos en parte, las razones por las que los lectores con bajo nivel de conocimientos previos se mostraron insensibles ante la presencia de textos con títulos, como también reflejaron los resultados de Wilhite. Respecto al segundo punto, debemos tener presente que los textos escolares se presentan bajo unas características muy heterogéneas que sobrepasan 


\section{4}

los casos estudiados y limitados a su ambigüedad. Por el contrario, los libros de textos varian en su complejidad y estructura, en la extensión y profundidad de los temas o en la adecuación de los contenidos a los niveles de conocimientos de los lectores. Ante tal diversidad, no puede predecirse el efecto que el título pueda producir en la comprensión. Como señalan Schwarz y Flammer (1981) los títulos potencian la codificación, almacenamiento y recuperación de la información leída en textos mínimamente estructurados, aunque presenten, como ya hemos aludido, alguna ambigüedad en sus planteamientos. Pero ante textos donde no se articula de una manera minimamente coherente las ideas que allí se expresen, sus efectos pueden resultar estériles.

\section{EN BUSCA DEL TITULO PERDIDO COMO TAREA INSTRUCCIONAL}

Lo comentado hasta ahora ha girado en torno a la mejora producida en la comprensión lectora cuando el título precede al contenido que se va a leer. Este efecto se ha explicado en base a la activación de un conocimiento previo relevante al tema textual, que orienta el procesamiento del texto. El papel del sujeto en estas situaciones se limita al papel de lector, en el sentido de que cumple su función al leer y procesar la información leída. Pero, ¿qué sucedería si el alumno o lector adoptara por un instante el papel del escritor y asumiera la responsabilidad de elaborar un título adecuado al pasaje que tiene delante de si?, ipodría resolver con éxito la tarea?, imejoraría la comprensión y retención de lo leído?

Según nos referíamos al final del apartado anterior, el éxito de esta empresa debe partir de dos presupuestos necesarios: que el nivel de conocimientos del lector se adecue al tópico del texto y que éste, a su vez, tenga una estructura minimamente organizada de las ideas que incluya. En tal caso, el lector podrá activar un esquema previo que le permita contextualizar y comprender el segmento leído. Debe seguidamente abstraer las ideas más importantes del pasaje (la macroestructura) y diferenciarlas de la información secundaria y complementaria (la microestructura), para finalmente, elegir de entre los títulos que aquellas ideas le sugieren, la que mejor represente toda la información leída. Todas estas fases suelen configurar una estrategia que sigue el sujeto competente, el sujeto adulto y experimentado frente al sujeto más inmaduro y menos competente. Una cuestión que resulta de extraordinario interés desde el punto de vista educativo es el considerar si esta habilidad, presente en los sujetos con una mayor pericia, resulta susceptible de ser implantada en lectores con una mẹnor competencia lectora a través de un programa de instrucción específico. En este sentido se han hecho algunos intentos serios al respecto. De hecho, la tarea de elaborar un título adecuado a un segmento del texto ha sido considerada como una actividad clave en la aplicación de diversos programas de instrucción encaminados a mejorar la comprensión lectora. En nuestro país, esta actividad ha formado parte de programas que han sido aplicados con éxito en los últimos cursos de la EGB (Sánchez, 1990) o en lo primeros cursos de BUP (León, 1991; León, Fdez-Pereira, Calvo y Picardo, 
en preparación). Incluso esta actividad forma parte de algunos procedimientos de estimulación de la comprensión lectora, especialmente indicado para sujetos con dificultades lectoras (Huerta y Matamala, 1990).

\section{Descripción de la tarea}

El principal objetivo que subyace a ésta actividad consiste en estimular al alumno a darse cuenta de la importancia que tiene el saber detectar la idea principal, el esquema general sobre el que gira el contenido de ese segmento del texto, siguiendo lo expuesto en el apartado teórico. En última instancia lo que se pretende con esta tarea es potenciar la comprensión, aprendiendo a captar mejor el significado del texto. Esta tarea le es de gran utilidad ya que le orienta hacia la detección de aquellos indicios claves del contenido del pasaje necesarios para estructurar la información esencial, o, viceversa, al identificar la relación capital y sus elementos principales puede, a través de ellos, elaborar el título correcto (e.g. características de..., causas que motivaron...).

El procedimiento de esta tarea suele ser simple. Una vez que los alumnos leen el texto como una actividad más dentro del aula (el pasaje ha sido seleccionado por el docente y obviamente se presenta sin ningún encabezamiento), se les anima a buscar el título que les sugiere el texto, tratando de responder a la cuestión "iqué título te sugiere este pasaje?" y anotando sus respuesta en un espacio reservado para ello. Sigamos el siguiente ejemplo, como si se tratase de uno de los textos seleccionados:

"Uno de los problemas con los que se encontró el imperio bizantino fue el de conseguir un producto muy preciado por aquella época: la seda. Sin embargo, el emperador Justiniano no deseaba comprárselo a los persas. Unos monjes llegados de la India y conocedores de este problema fueron a ver al soberano y le propusieron bacerse cargo de la fabricación de la seda $y$, de esta manera, evitar comprar tal mercancía. Ellos conocian como en una comarca llamada Serinda (cerca de la India) obtenian la seda por medio de gusanos. Una vez que tenía lugar la puesta de miles buevos y pasado un tiempo, se recubrian con estiércol y con un poco de calor provocaban su nacimiento. Una vez que esto ocurria, sólo bastaba alimentarlos con bojas de morera. El emperador exigió una prueba que confirmase tan sorprendente relato. Asi, los monjes volvieron a Serinda $y$ se trajeron buevos de estos gusanos a Bizancio. A partir de aquel momento, se les encangó la fabricación de la seda para aquel imperio".

En la medida en que los sujetos pertenecen a un mayor nivel académico, los alumnos suelen responder, después de varios intentos, con algún título más o menos sugerente. Sin embargo, conviene señalar que el título que se considere válido, debe ser preciso, debe ajustarse a lo referido en el texto. En otras palabras, el título seleccionado debe reflejar, más que el tema tratado a grandes rasgos, el contenido específico del pasaje. Un recurso válido para cumplir este objetivo consiste en presentarles varias opciones de títulos, que reflejan distintos niveles de abstracción. Siguiendo a Williams y cols. (1981), proponemos cuatro alternativas con las siguientes características: Una se dirige hacia el 


\section{6}

tema general, cuyo contenido abarca una información más amplia que la que se refiere el texto (e.g. el impero bizantino); una segunda opción corresponde al tema específico del pasaje (e.g. los inicios de la fabricación de la seda en Bizancio); en una tercera el título sugerido representa a una parte de la información complementaria (e.g. la comarca de Serinda); y por último, un cuarto título se relaciona con un tema anómalo que nada tenía que ver con el contenido del texto (e.g. las guerras médicas). La intromisión de esta tarea facilita la ejecución correcta de todos los alumnos, pero especialmente de los menos capaces. En la mayoría de los casos, la mayor dificultad estriba en discriminar entre el título general y específico.

\section{Cómo intervenir: El papel del docente}

El docente juega un papel clave en todo este proceso. De él parte su puesta en práctica y la instrucción de esta estrategia. Pero el éxito de su empresa no se garantiza por el mero hecho de implicarse en la tarea. En muchos casos conocemos sobradamente diferentes formas de enseñar y no con todas se obtienen los mismos resultados. Por este motivo, su actuación no debe limitarse a introducir su uso de una forma automática y rígida, siguiendo lo que podríamos denominar un entrenamiento ciego. En tal caso, más que informar a los alumnos sobre el porqué, cuándo, dónde y cómo debe utilizarse la estrategia y asumir la realización de este tipo de estrategias, es el profesor quien las lleva a cabo, realizando toda la tarea, resolviendo cualquier sospecha de problema. Si planteamos la tarea de esta manera estamos asumiendo el riesgo de convertir el dominio de la destreza en el fin de la instrucción. Por el contrario, esta habilidad debe entenderse como un medio para alcanzar un objetivo más crucial y relevante, aprender a captar el significado del texto.

Ante la situación descrita, cabe preguntarse qué hacer para entrenar a los alumnos en la mejora de la comprensión lectora, particularmente en aquellos cuyo rendimiento lector y comprensión es aún deficiente. Los datos de estudios relacionados con el tema revelan que los lectores menos competentes suelen presentar un conocimiento insuficiente sobre la utilidad y condiciones de empleo. Por ello, en un entrenamiento razonado, por contraste al ciego, se invita al alumno a desarrollar ciertas capacidades al mismo tiempo que se le informa de su finalidad y utilidad. Desde esta perspectiva se propone el uso de una instrucción directa porque aplica un conjunto de pasos secuenciados que conducen a la habilidad que se pretende transmitir. Entre éstos pasos se incluye la explicación y modelado así como distintos procedimientos designados para la corrección de errores. Este modelo de instrucción exige en el alumno un alto grado de participación ya que el apoyo del profesor se va diluyendo progresivamente a medida que se progresa en el programa.

El método de instrucción de ésta tarea se basa en la "instrucción razonada" de Brown y Palincsar (1984) que consta de tres fases: Explicación, modelado y práctica supervisada (véase para una revisión 
más ampliada Mateos, 1991, en un número anterior de esta misma revista). Con el primer paso, la explicación, se pretende informar de manera explícita a los alumnos sobre lo que se va a aprender, definiendo o describiendo los pasos a seguir y su utilidad. Con ello, se pretende que el alumno tome conciencia sobre el proceso, aumentando la posibilidad de que se actúe en consecuencia. Esta conciencia del proceso no debe limitarse a la exposición verbal del profesor, sino también mediante el diálogo y discusión con los alumnos. Con el modelado de los comportamientos lectores de los alumnos, se muestra paso a paso el procedimiento a seguir cuando se aplica una estrategia haciendo visible los procesos de pensamiento que normalmente se producen de forma encubierta durante la lectura. Finalmente, la última fase, consistente en una puesta en práctica bajo la supervisión del profesor, pretende lograr que el alumno practique el proceso observado. Esta actividad debe estar diseñada de modo que permita al alumno progresar a lo largo de un continuo de responsabilidad para lo cual el profesor debe ir retirando gradualmente el apoyo que proporciona hasta dejar el control del proceso en manos del alumno. Todo este proceso se fundamenta en las nociones de andamiaje de Bruner y en las ideas de Vygotski sobre la zona de desarrollo próximo, destacando la idea de que al principio sea el profesor el que asuma la mayor parte de responsabilidad y poco a poco ésta vaya pasando al alumno, bajo la supervisión del profesor.

Es muy importante que en el desarrollo de esta práctica, los diálogos no se orienten sólo en el "hacer" de la estrategia sino también en el razonamiento seguido por ellos durante su aplicación. Este punto es particularmente interesante ya que, no todos los alumnos entienden las explicaciones y demostraciones del profesor de manera idéntica ni al mismo tiempo, lo que podrá actuar de modo más ajustado a los problemas de aquellos, con explicaciones o demostraciones adicionales cuando las repuestas del alumno revelen una concepción o explicación erróneas. En este sentido, el profesor debe sugerir la discusión, potenciar la interacción profesor-alumno a través del diálogo y el razonamiento o justificación de esa elección, sugiriendo las vías que posibiliten la solución correcta más que facilitar directamente la respuesta correcta.

\section{A MODO DE CONCIUSION}

No ha sido nuestro objetivo presentar aquí un trabajo exhaustivo sobre los títulos, sino resaltar las ventajas que ofrece el aunar esfuerzos teóricos y aplicados en la consecución de un objetivo esencial común: comprender mejor lo que leemos. Los estudios súbre el título pueden ayudar a alcanzar este objetivo y para ello hemos partido desde dos perspectivas diferentes y estrechamente relacionadas. Por un lado, hemos analizado el concepto y la utilidad del título dentro del texto expositivo, en el sentido de que su presencia incrementa su comprensión y una mejor asimilación de la información leída por el lector. Por otro, hemos contemplado la posibilidad de enseñar al alumno en la búsqueda de un encabezamiento representativo de lo leído, como una útil estrategia lectora. Esta actividad puede resultar especialmente váli- 
da para aquellos lectores que presentan alguna dificultad comprensiva o una menor competencia lectora.

En cierto modo, podemos interpretar que elaborar un título supone activar o incluso generar un esquema que refleje de modo específico la información leída. La importancia de ésta tarea es que exige del lector comprender el texto leído y extraer la información más relevante, obligándole a desarrollar estrategias encaminadas a detectar las ideas principales que dan sentido a todo el texto y a discriminar la información macroestructural de la microestructural. Ahora bien, aunque el efecto de los títulos sea tan notorio, es preciso resaltar que los títulos deben estar correctamente elaborados, en el sentido de que deben representar la idea central sobre la que suele girar el texto. No hay que olvidar que la elaboración de un título en un material expositivo como un libro de texto, resulta frecuentemente una tarea costosa por la gran cantidad de conceptos y relaciones entre conceptos que deben tenerse en cuenta. Por otra parte, la información contenida en el texto debe tener un nivel adecuado a los conocimientos previos del lector. Ambos aspectos no siempre se tienen en cuenta en la composición escrita, lo que suele llevar parejo algunos déficits en la comprensión lectora.

El hecho de que este tipo de aplicaciones no se circunscriba a un determinado dominio o a un nivel académico específico supone una ventaja añadida a tener en cuenta en su aplicación en el aula. No debemos olvidar que se trata de una habilidad general, de una estrategia cognitiva de amplio espectro aplicable a un sinfín de materias que sobrepasan incluso a las actividades puramente académicas. De hecho, los trabajos realizados hasta ahora, los resultados han sido positivos tanto en los niveles académicos estudiados (EGB y BUP) como en las distintas materias en las que han sido aplicadas, tales como Sociales (León, 1991), Lengua y Literatura o Ciencias Naturales (León, Fernández, Calvo y Picardo, en preparación). Con todo, debe entenderse que las opciones descritas en el presente artículo son sólo algunas de las posibles y que, junto a otras, tratan de consolidar algunas de las funciones esenciales que debe cumplir un título para que, a través de él, podamos obtener un resultado óptimo de lo leído. Siguiendo a Brooks y Dansereau (1983) las funciones se resumirian en cuatro: a), desarrollar expectativas fiables en el lector sobre el contenido del pasaje; b), comprender las razones por las que cada título resulte apropiado para una determinada sección del texto; c), retener los títulos como si fuesen las llaves que abren las puertas del contenido que representan; y d), practicar el manejo de los títulos como una ayuda al recuerdo.

\section{Referencias}

ANDERSON, R.C. Y PICHTER, J.W. (1978). "Recall of previously unrecallabre information following a shift in perspective". Jourmal of Verbal Learning and Verbal Bebavior, 17, 1-12.

BRANSFORD, J.D. Y JOHNSON, M.K (1972). "Contextual prerequisites for understanding of comprehension and recall". Joumal of Verbal Learning and Verbal Bebavior, 11, 717-726.

Brooks, L.W., DANSERAU, D. F., Spurun, J.E. y Houry, C.D. (1983). "Effects of headings on text processing". Joumal of Educational Psycbology, 75, 2, 292-302. 
Brown, A. L. PAUNCSAR, A. S. Y ARMBruSTER, B. B. (1984). Instructing comprehension-fostering activities in interactive learning situations. En H. Mandl, N. L. Stein y T. Trabasso (Eds), Leaming and comprebension of text. Hillsdale, Nueva Jersey: Erlbaum.

Dooung, D. J. Y LACruan, R. (1971). Effects of comprehension on retention of prose. Journal of Experimental Psychology, 88, 216-222.

GRANT, R. y DAVEY, B. (1991). "How do headings affect text-processing?". Reading Research and Instruction, 31, (1), 12-21.

Huerta, E. y Matamala, A. (1990). Programa de estimulación de la comprensión lectora. 3 vol. Madrid: Visor.

Jonassen, D., HARTIEY, J. y Trueman, M. (1986). "The effects of learner-generated versus text-provided headings on immediate and delayed recall and comprehension: an exploratory study". Human Leaming, 5, 139-150.

KuzMINSKr, E. (1977). "Altering comprehension: the effects of biasing titles on text comprehension". Memony and Cognition, 5, 482-490.

LEON, J.A. (1991). "Intervención en estrategias de comprensión: un modelo basado en el conocimiento y aplicación de la estructura del texto". Infancia y Aprendizaje, 56, 77-91.

Leon, J.A., Fdez-PererRa, C., Calvo, S y Picardo, C. (en preparación). Un modelo de intervención en el aula: Aplicaciones sobre areas de Lengua y Biologia.

MATEos, M. (1991). “Instrucción directa en estrategias de comprensión lectora”. Comunicación, Lenguaje y Educación, 9, 89-95.

SANCHEZ, E. (1990). "Estructuras textuales y procesos de comprensión: un programa para instruir en la comprensión de textos". Estudios de Psicologia, 41, 21-40.

SCHWARZ, M. Y Flammer, A. (1981). "Text structure and title - effects on comprehension and recall". Joumal of Verbal Leaming and Verbal Bebavior, 20, 61-66.

SPYRIDAkns, J.H. Y StaNDAL, T.C. (1987). "Signals in expository prose: Effects on reading comprehension". Reading Research Quarterly, 22, 283-295.

WILHITE, S. C. (1988). "Reading for a multiple-choice test: Headings as schema activators". Joumal of Reading Bebavior, 20, 215-228.

WILHITE, S. C. (1989). "Headings as memory facilitators: the importance of prior knowledge". Journal of Educational Psychology, 81, 1, 115-117.

WHUAMS, J.P., TAYLOR, M.B. Y GANGER, S. (1981). "Text variations at the level of the individual sentence and the comprehension of simple Expository Paragraphs". Journal of Educational Psychology, 73, 6, 851-865. 
El título como recurso didáctico Jose Antonio León y Angel Martín Ginard CL\&E, 1993, 19-20, 159-170

Resumen: Este trabajo incide en el estudio de los títulos desde una doble perspectiva. Por un lado, presenta algunos trabajos teóricos y empíricos que explican y avalan de modo experimental la mejora que los títulos ejercen sobre la comprensión lectora. Por otro, analiza las ventajas que ofrece el instruir a los alumnos de muy diferentes niveles en la elaboración de títulos como una estrategia de comprensión

Datos sobre el autor: Los autores trabajan en el Departamento de Psicología Básica de la Facultad de Psicologia perteneciente a la Universidad Autónoma de Madrid

Dirección: Crta. de Colmenar, km. 15. Cantoblanco. 28049 Madrid

Agradecimientos: Esta revisión forma parte de diversas investigaciones que fueron en su momento financiadas por el CIDE en la convocatoria de 1990. Asimismo, deseamos agra decer a Charo Serrano Collantes su colaboración en la elaboración de este manuscrito

(C) De todos los artículos deberá solicitarse por escrito autorización de CL\&E y de los autores para el uso en forma de facsímil, fotocopia o cualquier otro medio de reproducción impresa. CL\&E se reserva el derecho de interponer acciones legales necesarias en aquellos casos en que se contravenga la ley de derechos de autor.

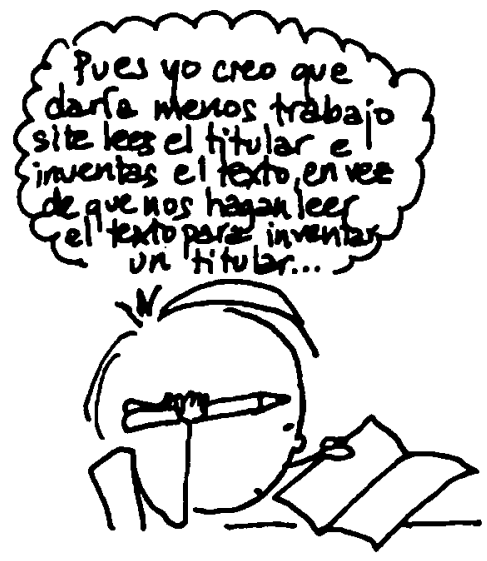

